\title{
Mercaptopurine Oral Suspension
}

National Cancer Institute

\section{Source}

National Cancer Institute. Mercaptopurine Oral Suspension. NCI Thesaurus. Code C111757.

An oral suspension containing the thiopurine-derivative antimetabolite 6-mercaptopurine, with potential antineoplastic activity. Upon oral administration, mercaptopurine is metabolized by hypoxanthine-guanine phosphoribosyltransferase (HGPRT ase) to its active metabolite 6-thioinosine monophosphate (TIMP); TIMP inhibits nucleotide interconversions and de novo purine ribonucleotide synthesis, which both blocks the formation of purine nucleotides and inhibits DNA synthesis. This agent is also incorporated into DNA in the form of deoxythioguanosine, which results in the disruption of DNA replication. By blocking DNA synthesis and replication, cancer cells are unable to proliferate. Compared to the tablet formulation, the liquid is easier to swallow and offers more flexibility and accuracy in dosing, which is beneficial for use in pediatric patients. 